\title{
Host genetic factors in susceptibility to mycobacterial disease
}

\author{
Authors: Christopher JA Duncan ${ }^{A}$ and Sophie Hambleton ${ }^{B}$
}

\begin{abstract}
Primary immunodeficiencies (PID) are a group of rare inherited disorders that manifest as heightened susceptibility to infection, autoimmunity and/or malignancy. By exploring their genetic and cellular aetiology, we can learn much about the basis of pathogen-specific immunity in humans. This is exemplified by mycobacterial susceptibility, which occurs across several types of PID, either as an isolated problem or as part of a broader pattern of susceptibility to infection. These experiments of nature have contributed to our understanding of the central role of T cells in activating infected macrophages to eliminate phagosomal mycobacteria through mutually activating, cytokine-dependent interactions. In recent years, the discovery of novel forms of PID has emphasised the important role of dendritic cells and monocytes in mycobacterial defence in humans. Here, we provide a brief overview of these new disorders alongside other genetic causes of susceptibility to mycobacterial disease.
\end{abstract}

KEYWORDS: Mycobacteria, tuberculosis, BCG, primary immunodeficiency, human genetics, dendritic cell

\section{Mycobacterial disease}

Mycobacterium spp. are environmentally ubiquitous bacilli responsible for a significant burden of global disease. A spectrum of virulence runs across the genus: Mycobacterium tuberculosis (MTB) kills approximately 1.3 million people per annum, whereas Mycobacterium leprae continues to cause disfiguring disease. The less pathogenic non-tuberculous Mycobacteria (NTM) are mainly opportunistic pathogens of patients with structural lung disease or immunodeficiency; similarly, the live-attenuated vaccine strain of Mycobacterium bovis Bacille Calmette-Guérin (BCG) rarely causes

Authors: ${ }^{\text {p }}$ rofessor of paediatrics and immunology, Institute of Cellular Medicine, Newcastle University, Newcasle-upon-Tyne, UK, and honorary consultant paediatrician, Great North Children's Hospital, Newcastle-upon-Tyne, UK; B academic clinical lecturer in infectious diseases, Institute of Cellular Medicine, Newcastle University, Newcastle-upon-Tyne, UK, and Department of Infection and Tropical Medicine Royal Victoria Infirmary, Newcastle-uponTyne, UK disseminated disease (termed 'BCG-osis' ${ }^{1}$ ) in the absence of immunodeficiency.

\section{Mycobacterial pathogenesis}

Insights into mycobacterial pathogenesis have come mostly from animal models and patient studies and, although we have a broad concept of pathogenic mechanisms, significant gaps remain (reviewed in Philips and Ernst ${ }^{2}$ ). Macrophages are the archetypal target cell for Mycobacterium spp., although other phagocytes (including neutrophils, monocytes and dendritic cells (DC)) also ingest bacilli ${ }^{2}$ and can support replication. ${ }^{3}$ Uptake is conditional for mycobacterial virulence and mycobacteria take advantage of considerable redundancy in the process of phagocytosis to enter target cells. Mycobacteria survive and replicate within phagosomes, autophagosomes and possibly also the cytosol ${ }^{2}$ of macrophages. The classical T helper $1\left(\mathrm{~T}_{\mathrm{H}} 1\right)$ cytokines, interferon- $\gamma($ IFN- $\gamma)$ and tumour necrosis factor- $\alpha$ (TNF- $\alpha$ ), produced by interleukin (IL)-12polarised CD4 $+\mathrm{T}$ cells are required to activate macrophages and enable mycobacterial killing within the phagosome. ${ }^{2}$ The role of antigen-presenting cells in this process lacks clear definition (see below), although MTB has multiple mechanisms for downregulating major histocompatibility complex (MHC) class II expression in infected cells, ${ }^{4}$ suggesting that evasion of antigen presentation to CD4+ T cells is advantageous.

\section{Mycobacterial susceptibility}

Around ten times more people are infected with MTB than develop clinical tuberculosis (active TB), proving that the immune system, although unable to eradicate infection, often contains it sufficiently to prevent disease. Mycobacterial susceptibility is a feature of a range of clinical immunodeficiency syndromes. For example, the annual risk of active TB in patients with acquired immunodeficiency syndrome (AIDS) because of human immunodeficiency virus (HIV-1) infection is the same as the lifetime risk in the immunocompetent. ${ }^{5}$ Heightened susceptibility to mycobacterial disease is also a feature of several types of primary immunodeficiency (PID) caused by single-gene defects in immune function. Reflecting the frequency with which different Mycobacterium spp. are encountered, these disorders typically present not with MTB but with disease caused by its less pathogenic relatives, NTM and BCG. Efforts to define the molecular basis of these disorders have taught us important 
lessons about human mycobacterial immunity, including the crucial role of T cells, dendritic cells and the IL-12/IL$23-$ IFN- $\gamma$-signal transducer and activator of transcription 1 (STAT1) circuit.

\section{Aims and scope}

In this review, we summarise data on monogenic disorders that confer enhanced susceptibility to mycobacterial disease, with a particular focus on the recently described dendritic cell deficiency syndromes, and reflect on the fundamental insights into the nature of protective mycobacterial immunity that these 'experiments of nature' provide. Population-based genetic studies of single nucleotide variants (also known as single nucleotide polymorphisms) linked to mycobacterial susceptibility and data from murine models are outside the scope of this article.

\section{Single gene factors in mycobacterial disease susceptibility}

The analysis of monogenic immune defects has been a rich source of information about non-redundant cell types and molecular pathways in the human immune response to pathogens, ${ }^{6}$ by turns validating ${ }^{7}$ or challenging ${ }^{8}$ findings in animal models. Gene defects have been elucidated by various means, including linkage analysis, candidate gene studies and unbiased approaches, such as whole-exome sequencing.

Mycobacterial disease occurs as part of a broader pattern of susceptibility to infection in several forms of PID. The central role of T cells is illustrated by the susceptibility of patients with severe combined immunodeficiency to disseminated BCG disease, among many other infections. ${ }^{9}$ Chronic granulomatous disease (CGD), a monogenic disorder of the oxidative burst resulting from several mutations in NADPH oxidase, ${ }^{10}$ produces vulnerability to a specific range of intracellular microorganisms (including Mycobacterium spp. in patients from endemic regions), ${ }^{11}$ reinforcing the importance of intraphagosomal killing in the effective control of mycobacterial infection.

Another class of PID to inform our understanding of mycobacterial immunity is the rare Mendelian susceptibility to mycobacterial disease (MSMD) (reviewed in Casanova and $\mathrm{Abel}^{12}$ ). In the western world, most of these patients present with BCG-osis or NTM disease (although also at increased risk of TB). In contrast to classic forms of PID, patients with MSMD are often otherwise healthy, despite some increased susceptibility to other intramacrophagic pathogens (including disseminated non-typhoidal salmonella). Candidate gene studies led to the discovery of several autosomal recessive and $\mathrm{X}$-linked inherited gene variants in the classic $\mathrm{T}_{\mathrm{H}} 1$ circuit: IL-12 p40 subunit (IL12B), and IL-12 receptor beta-subunit (IL12RB1), interferon- $\gamma$ receptor 1 (IFNGR1) and interferon- $\gamma$ receptor 2 (IFNGR2) and STAT1. These lesions are believed to disrupt the mutually activating interaction between the macrophage and $\mathrm{T}$ cell that culminates in the engagement of intraphagosomal killing mechanisms.

Although often considered as a form of MSMD, PID associated with mutations of nuclear factor-kappa B-essential modulator (NEMO) or IKBA (NFKBIA) typically produces a broader infectious phenotype (reviewed by a number of authors $\left.{ }^{12-15}\right)$. Therefore, it is all the more striking that iatrogenic blockade of the same pathway with anti-TNF to treat autoimmune disease is a well-documented cause of TB reactivation. More recently, MSMD-associated variants have been identified in $C Y B B^{16}$ and interferon-stimulated gene 15 (ISG15). ${ }^{17}$ Although CYBB is the gene classically mutated in $\mathrm{X}$-linked CGD, Bustamante et al identified MSMD kindreds with distinct allelic variants that led to a milder, macrophagerestricted defect in the oxidative burst. ${ }^{16}$ More intriguingly, although ISG15 is necessary for antiviral immunity in mice, ${ }^{18}$ the reported human phenotype displayed preserved antiviral immunity and a specific defect in the IFN- $\gamma$ response to mycobacteria, ${ }^{17}$ reinforcing the concept that monogenic immune defects in humans and mice can have divergent effects. It is likely that many more relevant mycobacterial susceptibility variants exist and will be identified in the coming years because up to half of patients with MSMD do not have a molecular diagnosis. $^{14}$

\section{DC deficiency in mycobacterial susceptibility}

Dendritic cells (DC) are specialised antigen-presenting cells of the mononuclear phagocyte system that have unique functional capabilities, including lymph node antigen trafficking and potent activation of naïve T cells. ${ }^{19}$ In capturing, processing and presenting antigen to T cells, DC are the main conduit between the innate and adaptive immune systems and, therefore, their role in the human immune system has been viewed as central. Until recently, no DC deficiency syndromes had been discovered to test this assumption. Functional defects in antigen presentation have been described; for example, MHC class II deficiency (known as bare lymphocyte syndrome) or Wiskott-Aldrich syndrome (which causes a defect in immune synapse formation). In addition, numerical DC deficiency is a feature of PIDs characterised by broad cytopenias (eg reticular dysgenesis, Ikaros deficiency and C-X-C chemokine receptor type 4 (CXCR4) deficiency (WHIM syndrome)). However, none of these conditions inform the specific role of the DC. Below, we describe the molecular basis and characteristics of three recently discovered monogenic DC deficiencies that are associated with mycobacterial susceptibility. To put these findings into context, we first summarise human DC development.

\section{DC development in humans}

Understanding of the development and functional specialisation of human DC lags behind that of the mouse. Ethical constraints mean that most human studies are performed in peripheral blood or using in vitro monocytederived DC systems that might have limited relevance to tissue in vivo. There is also limited consistency of cell surface markers used to define subpopulations of DC across mouse and human. As such, it is difficult to infer from mouse the facts of human DC development.

With the probable exception of self-renewing skin-resident Langerhans cells and some central nervous system resident mononuclear phagocytes, human DC subsets (including various subsets of myeloid DC, and plasmacytoid DC; Table 1) are derived from haematopoietic progenitor cells (both granulocyte-macrophage progenitors and multilymphoid 
Table 1. Human dendritic cell subtypes. ${ }^{a}$

\begin{tabular}{ll}
$\begin{array}{ll}\text { Group } \\
\text { Myeloid DC }\end{array}$ & $\begin{array}{l}\text { Subset } \\
\text { Major }(C D 1 C+) \\
\text { Cross-presenting }(C D 141+)\end{array}$ \\
Plasmacytoid DC $\left(C D 123^{+}\right)$ & CD14+ DC \\
Monocyte-related DC & CD16+ monocyte \\
& Inflammatory DC \\
\hline${ }^{a}$ Adapted from Collin et al ${ }^{19} \cdot \mathrm{DC}=$ dendritic cell.
\end{tabular}

progenitors $^{20}$ ) in the bone marrow. ${ }^{19}$ This conclusion is supported by data from haematopoietic stem cell transplantation (HSCT) in humans. ${ }^{21}$

There is no evidence that monocytes differentiate into DC in the steady state in humans and it is currently unclear what relation blood DC subsets have to those in tissues ${ }^{19,22}$ (Table 1). Committed DC precursors have not been found in either blood or bone marrow and it appears that tissue DC are derived from blood DC and/or CD34+ progenitor cells in the blood. ${ }^{19}$ In this context, in natura human DC deficiency can also teach us about DC development.

\section{DC deficiency}

Historically, DC numbers were not measured in clinical diagnostic laboratories and this might account for the delayed recognition of DC deficiency syndromes in practice (associated monocytopenia is a hint). Novel algorithms aid DC profiling in peripheral blood ${ }^{23}$ and can lead to more efficient recognition of DC deficiency. There are currently three recognised genetic DC deficiency syndromes (Table 2), which are characterised by deficiency of DC subsets in the tissues and/or peripheral blood together with other haematological and clinical features. ${ }^{22}$

\section{Table 2. Dendritic cell deficiency syndromes.}

\begin{tabular}{|c|c|c|c|}
\hline \multirow[t]{2}{*}{ Feature } & \multicolumn{3}{|c|}{ Syndrome } \\
\hline & DCML & IRF8 (K108E) & IRF8 (T80A) \\
\hline $\mathrm{pDC}$ & Absent & Absent & Normal \\
\hline$C D 11 c+m D C$ & Absent & Absent & Normal \\
\hline $\mathrm{CD} 1 \mathrm{c}+\mathrm{mDC}$ & Absent & Absent & Reduced \\
\hline CD141+ mDC & Absent & Absent & Normal \\
\hline Monocytes & Reduced & Reduced & Normal \\
\hline GMPs & Reduced & Increased & Not tested \\
\hline MLPs & Absent & Increased & Not tested \\
\hline Age & $7-60$ years & $<3$ months & $<2$ years \\
\hline Inheritance & AD/de novo & $A R$ & $A D$ \\
\hline \multicolumn{4}{|c|}{$\begin{array}{l}{ }^{a} \text { Adapted from Collin et al. }{ }^{22} \mathrm{AD}=\text { autosomal dominant; } \mathrm{AR}=\text { autosomal } \\
\text { recessive; } \mathrm{DCML}=\text { dendritic cell, monocyte, } \mathrm{B} \text { and NK lymphoid; GMPs = } \\
\text { granulocyte myeloid precursors; } \mathrm{IFR} 8=\text { interferon regulatory factor } 8 ; \mathrm{mDC}= \\
\text { myeloid dendritic cell; } \mathrm{pDC}=\text { plasmacytoid dendritic cell; MLPs = myeloid- } \\
\text { lymphocyte precursors. }\end{array}$} \\
\hline
\end{tabular}

\section{DCML because of GATA2 mutation}

The most frequently encountered DC deficiency syndrome to date is so-called DC, monocyte, B and NK lymphoid deficiency (Dendritic cell, monocyte, B and NK lymphoid $(\mathrm{DCML})$ deficiency $^{24}$ ) resulting from autosomal dominant mutations in GATA-binding factor 2 (GATA2). ${ }^{25,26}$ This broad mononuclear cell deficiency has also been labelled 'MonoMAC syndrome' because of its association of heightened susceptibility to NTM infection (specifically M. avium complex (MAC)) with autosomal dominant or sporadic monocytopenia. ${ }^{27}$ This is a progressive disorder and, by the time mycobacterial susceptibility is evident, blood and tissue DC are typically absent, although macrophages and Langerhans cells are preserved. ${ }^{28}$ Associated features might include susceptibility to viral and fungal infections, autoimmunity, lymphoedema, myelodysplasia that might transform to acute myeloid leukaemia and pulmonary alveolar proteinosis (a rare disorder of alveolar macrophage function leading to alveolar protein accumulation and respiratory failure). ${ }^{28,29}$

Candidate gene studies ${ }^{26}$ and exome sequencing ${ }^{25}$ conclusively established that DCML/MonoMAC is caused by heterozygous mutation of the transcriptional regulator GATA2. Haploinsufficiency of this gene impairs normal haematopoietic development, resulting in dramatic loss of multilymphoid progenitors and partial loss of granulocyte-macrophage progenitors in bone marrow. ${ }^{24}$ Despite this, most patients are healthy in their first one to two decades and do not experience problems with BCG. It has been proposed that preserved immune cell numbers (granulocytes, T lymphocytes, plasma cells and class-switched B cells together with tissue macrophages and dermal Langerhans cells) confer sufficient immunity to deal with most childhood infections. ${ }^{22}$ It perhaps also implies a redundant role for DC in antigen presentation ${ }^{30}$ and detailed analysis of the function and specificity of CD4+ T cells in patients with DCML will help to clarify the degree of antigen presentation defect(s) and their consequences. Many patients with GATA2 deficiency are susceptible to mycobacterial disease (particularly NTM) and their cells exhibit defective IL-12 and IFN- $\gamma$ responses to toll-like receptor (TLR) agonists in vitro. ${ }^{24}$ However, the precise contribution of specific cell types (eg DC versus monocytes) is impossible to dissect.

\section{DC deficiency resulting from IRF8 mutations}

The discovery of two distinct DC deficiency syndromes resulting from mutations in IFN-regulatory factor $8(I R F 8)^{7}$ further clarifies the role of DC in mycobacterial immunity. The IFN regulatory factors are transcriptional regulators involved in haematopoiesis as well as in the response to IFN and TLR signalling leading to $I L 12 B$ and nitric oxide synthase 2 (NOS2) transactivation; ${ }^{31}$ murine studies have shown Irf 8 to be central to DC development. ${ }^{32}$ In humans, two mutations have been described in the IRF8 DNA-binding domain ${ }^{7}$ (Table 2): the AR mutation K108E caused an isolated severe deficiency of DC and monocytes with associated myeloproliferation and profound immunodeficiency requiring HSCT; the AD mutation T80A was instead associated with MSMD and an apparently specific deficiency of circulating CD11c+CD1c+ myeloid DC. ${ }^{7}$ The latter syndrome seems to offer an important test of the role of DC in mycobacterial immunity, but poses several 
questions. Perplexingly, the 'missing' DC subset is not that typically associated with cross-presentation and neither does it correspond to the CD8 $\alpha$ DC subset that is preferentially lost in murine Irf8 deficiency. Furthermore, BCG responsiveness was surprisingly preserved in patient peripheral blood ex vivo, although a defect in IL-12 production in response to TLR7/8 stimulation was observed in patients with AD T80A compared with controls. One interpretation is that mycobacterial susceptibility in AD T80A is the result of failure of IL-12 production specifically by this CD11c+CD1c+ DC subset, similar to defects in IL12p40 or IL12R (see above). Perhaps in keeping with this, CD1c-restricted T cells with mycobacterial specificity have been reported ${ }^{33}$ and might require $\mathrm{CD} 1 \mathrm{c}+$ myeloid DC for their development and priming. The impact of AD IRF8 deficiency on mycobacterial killing within infected phagocytes merits further investigation as another potential mechanism of increased susceptibility.

\section{Perspectives on DC development and function}

The relative preservation of tissue macrophages and Langerhans cells in the face of the profound monocytopenia that accompanies deficiencies of GATA2 and AR IRF8 implies a distinct developmental origin for these cells. ${ }^{22}$ Although both disorders impair DC development in the bone marrow, the accompanying context differs dramatically: the K108E mutant results in accumulation of myeloid-lymphocyte precursors (MLP) and granulocyte-myeloid precursors (GMP), ${ }^{7}$ whereas GATA2 deficiency causes a marked deficit of MLP (and to a lesser degree GMP). ${ }^{24}$ This indicates that these molecules function at distinct points in DC differentiation, with GATA2 acting proximally and IRF8 distally in the MLP/GMP stage. Interestingly, CD4+ T cells from the patient with AR IRF8 deficiency exhibited impaired production of various cytokines (including IFN- $\gamma$ and IL-17) to CD3/CD28 stimulation ex vivo, suggesting a functional $\mathrm{T}$ cell defect in vivo. ${ }^{7}$ In keeping with this interpretation, an unusually high proportion of naïve cells in the T cell compartment of this patient was observed. ${ }^{7}$ Further studies to define the T cell phenotype in both DC deficiency syndromes will shed light on the possible effects of impaired antigen presentation on the $\mathrm{T}$ cell repertoire.

\section{Summary}

The identification of DC deficiency in the context of mycobacterial susceptibility adds a missing piece to the
Fig 1. Model of mycobacterial susceptibility. Single gene variants in the molecular components in red type are implicated in mycobacterial susceptibility in humans. In addition, numerical or functional cellular deficiency syndromes linked to mycobacterial susceptibility are outlined below the relevant cell type. CGD = chronic granulomatous disease; $\mathrm{DCML}=$ dendritic cell, monocyte, B and NK lymphoid deficiency; IFN = interfeuron; IL = interleukin; IRF8 $=$ interferon-regulatory factor 8 ; ISG15 $=$ interferon-stimulated gene 15 ; NEMO = nuclear factorkappa B-essential modulator; $\mathrm{SCID}=$ severe combined immunodeficiency syndrome; STAT = signal transducer and activator of transcription 1 .

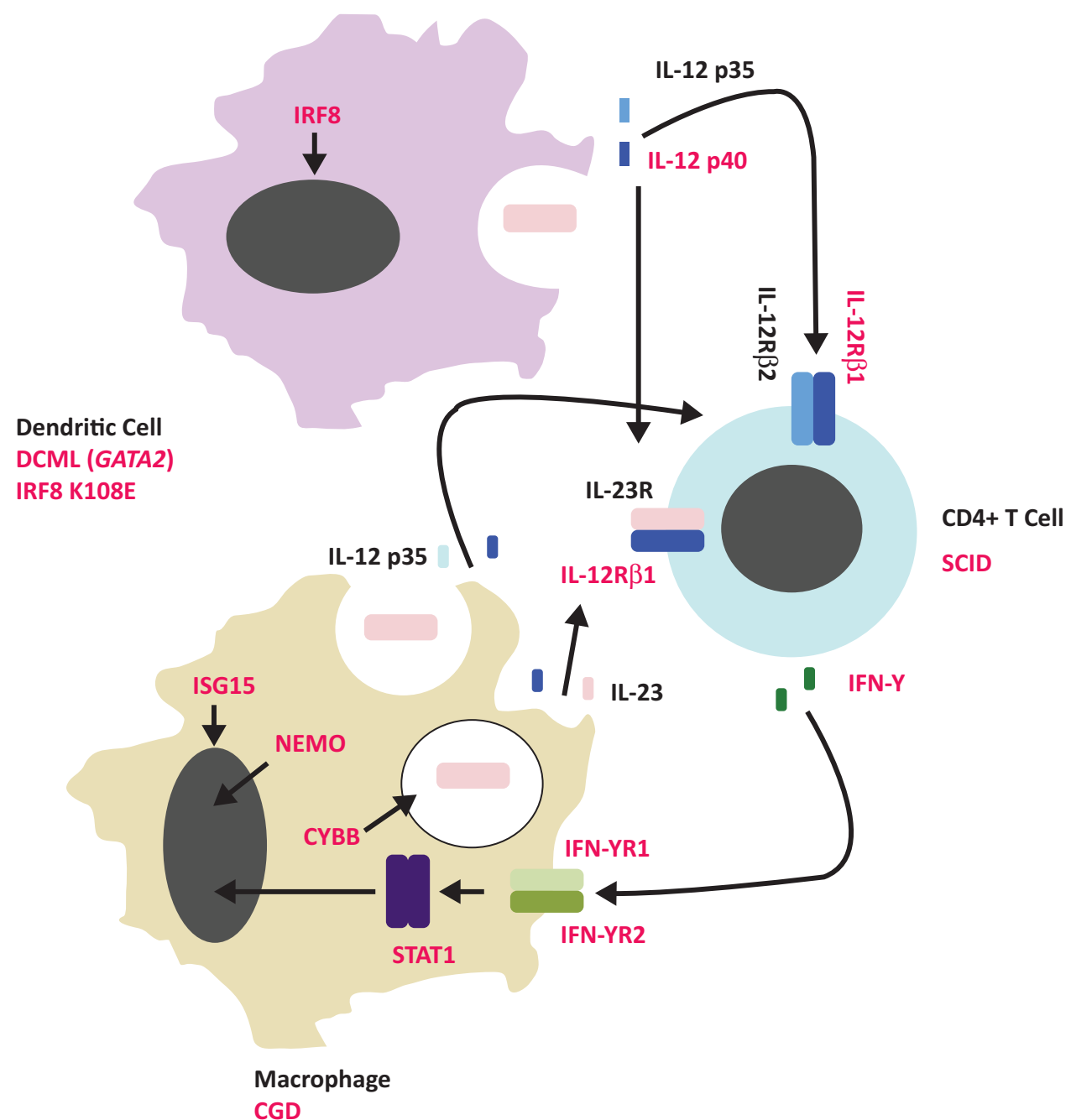


jigsaw of pathogen-specific immunity in humans (Fig 1). To the conventional diagrammatic representation of the mycobacterially infected macrophage communing with an antigen-specific T lymphocyte, we must add a third player: the DC. Future studies in these rare patients should seek to disentangle the respective contributions of DC, monocyte and $\mathrm{T}$ cell to functional immunodeficiency and could inform attempts to bolster adaptive immunity by smarter approaches to vaccination.

\section{References}

1 Salem S, Gros P. Genetic determinants of susceptibility to mycobacterial infections: IRF8, a new kid on the block. Adv Exp Med Biol 2013;783:45-80.

2 Philips JA, Ernst JD. Tuberculosis pathogenesis and immunity. Annu Rev Pathol 2012;7:353-84.

3 Wolf AJ, Linas B, Trevejo-Nunez GJ et al. Mycobacterium tuberculosis infects dendritic cells with high frequency and impairs their function in vivo. J Immunol 2007;179:2509-19.

4 Harding CV, Boom WH. Regulation of antigen presentation by Mycobacterium tuberculosis: a role for Toll-like receptors. Nat Rev Microbiol 2010;8:296-307.

5 McShane H. Co-infection with HIV and TB: double trouble. Int $J$ STD AIDS 2005;16:95-100.

6 Casanova JL, Abel L. Primary immunodeficiencies: a field in its infancy. Science 2007;317:617-9.

7 Hambleton S, Salem S, Bustamante J et al. IRF8 mutations and human dendritic-cell immunodeficiency. $N$ Engl J Med 2011;365:127-38.

8 Hambleton S, Goodbourn S, Young DF et al. STAT2 deficiency and susceptibility to viral illness in humans. Proc Natl Acad Sci USA 2013;110:3053-8.

9 Aloj G, Giardino G, Valentino L et al. Severe combined immunodeficiences: new and old scenarios. Int Rev Immunol 2012;31:43-65.

10 Holland SM. Chronic granulomatous disease. Hematol Oncol Clin North Am 2013;27:89-99

11 Abel L, El-Baghdadi J, Bousfiha AA, Casanova JL, Schurr E. Human genetics of tuberculosis: a long and winding road. Philos Trans $R$ Soc Lond B Biol Sci 2014;369:20130428.

12 Casanova JL, Abel L. Genetic dissection of immunity to mycobacteria: the human model. Annu Rev Immunol 2002;20:581-620.

13 Fortin A, Abel L, Casanova JL, Gros P. Host genetics of mycobacterial diseases in mice and men: forward genetic studies of BCGosis and tuberculosis. Annu Rev Genomics Hum Genet 2007;8:163-92.

14 Al-Muhsen S, Casanova JL. The genetic heterogeneity of mendelian susceptibility to mycobacterial diseases. J Allergy Clin Immunol 2008;122:1043-51.

15 Cottle LE. Mendelian susceptibility to mycobacterial disease. Clin Genet 2011;79:17-22.

16 Bustamante J, Arias AA, Vogt G et al. Germline CYBB mutations that selectively affect macrophages in kindreds with X-linked predisposition to tuberculous mycobacterial disease. Nat Immunol 2011;12:213-21.

17 Bogunovic D, Byun M, Durfee LA et al. Mycobacterial disease and impaired IFN-gamma immunity in humans with inherited ISG15 deficiency. Science 2012;337:1684-8.
18 Skaug B, Chen ZJ. Emerging role of ISG15 in antiviral immunity. Cell 2010;143:187-90.

19 Collin M, McGovern N, Haniffa M. Human dendritic cell subsets. Immunology 2013;140:22-30.

20 Doulatov S, Notta F, Eppert K et al. Revised map of the human progenitor hierarchy shows the origin of macrophages and dendritic cells in early lymphoid development. Nat Immunol 2010;11:585-93.

21 Haniffa M, Ginhoux F, Wang XN et al. Differential rates of replacement of human dermal dendritic cells and macrophages during hematopoietic stem cell transplantation. J Exp Med 2009;206:37185 .

22 Collin M, Bigley V, Haniffa M, Hambleton S. Human dendritic cell deficiency: the missing ID? Nat Rev Immunol 2011;11:575-83.

23 Jardine L, Barge D, Ames-Draycott A et al. Rapid detection of dendritic cell and monocyte disorders using $\mathrm{CD} 4$ as a lineage marker of the human peripheral blood antigen-presenting cell compartment. Front Immunol 2013;4:495.

24 Bigley V, Haniffa M, Doulatov S et al. The human syndrome of dendritic cell, monocyte, B and NK lymphoid deficiency. J Exp Med 2011;208:227-34.

25 Dickinson RE, Griffin H, Bigley V et al. Exome sequencing identifies GATA-2 mutation as the cause of dendritic cell, monocyte, B and NK lymphoid deficiency. Blood 2011;118:2656-8.

26 Hsu AP, Sampaio EP, Khan J et al. Mutations in GATA2 are associated with the autosomal dominant and sporadic monocytopenia and mycobacterial infection (MonoMAC) syndrome. Blood 2011;118:2653-5.

27 Vinh DC, Patel SY, Uzel G et al. Autosomal dominant and sporadic monocytopenia with susceptibility to mycobacteria, fungi, papillomaviruses, and myelodysplasia. Blood 2010;115:1519-29.

28 Dickinson RE, Milne P, Jardine L et al. The evolution of cellular deficiency in GATA2 mutation. Blood 2014;123:863-74.

29 Spinner MA, Sanchez LA, Hsu AP et al. GATA2 deficiency: a protean disorder of hematopoiesis, lymphatics, and immunity. Blood 2014;123:809-21.

30 Hume DA. Macrophages as APC and the dendritic cell myth. $J$ Immunol 2008;181:5829-35.

31 Tamura T, Yanai H, Savitsky D, Taniguchi T. The IRF family transcription factors in immunity and oncogenesis. Annu Rev Immunol 2008;26:535-84.

32 Schiavoni G, Mattei F, Sestili P et al. ICSBP is essential for the development of mouse type I interferon-producing cells and for the generation and activation of CD8alpha(+) dendritic cells. J Exp Med 2002;196:1415-25.

33 Ulrichs T, Moody DB, Grant E, Kaufmann SH, Porcelli SA. T-cell responses to CD1-presented lipid antigens in humans with Mycobacterium tuberculosis infection. Infect Immun 2003;71:3076-87.

Address for correspondence: Prof S Hambleton, Primary Immunodeficiency Group, Institute of Cellular Medicine, 4th Floor Catherine Cookson Building, Newcastle University Medical School, Framlington Place, Newcastle-upon-Tyne, NE2 4HH, UK.

Email: sophie.hambleton@ncl.ac.uk 